\title{
Emotional Modulation of Pain: Is It the Sensation or What We Recall?
}

\author{
Fabio Godinho, ${ }^{1,2}$ Michel Magnin, ${ }^{1}$ Maud Frot, ${ }^{1}$ Caroline Perchet, ${ }^{1}$ and Luis Garcia-Larrea ${ }^{1}$ \\ ${ }^{1}$ Institut National de la Santé et de la Recherche Médicale (INSERM), Equipe Mixte INSERM 342, Central Integration of Pain, and ${ }^{2}$ Department of \\ Functional Neurosurgery, Unité 400, Hôpital Neurologique Pierre Wertheimer, 69003 Lyon, France
}

\begin{abstract}
Emotions modulate pain perception, although the mechanisms underlying this phenomenon remain unclear. In this study, we show that intensity reports significantly increased when painful stimuli were concomitant to images showing human pain, whereas pictures with identical emotional values but without somatic content failed to modulate pain. Early somatosensory responses ( $<200 \mathrm{~ms})$ remained unmodified by emotions. Conversely, late responses showed a significant enhancement associated with increased pain ratings, localized to the right prefrontal, right temporo-occipital junction, and right temporal pole. In contrast to selective attention, which enhances pain ratings by increasing sensory gain, emotions triggered by seeing other people's pain did not alter processing in SI-SII (primary and second somatosensory areas), but may have biased the transfer to, and the representation of pain in short-term memory buffers (prefrontal), as well as the affective assignment to this representation (temporal pole). Memory encoding and recall, rather than sensory processing, appear to be modulated by empathy with others' physical suffering.
\end{abstract}

Key words: attention; emotion; pain; electrophysiology; empathy; prefrontal

\section{Introduction}

Empathy has been recognized as an evolved function, with precursors such as mimicry and imitation. Fundamental to this construct is that, unless inhibited, the perception of another person's behavior activates representations of the personal experiences associated with that behavior (Goubert et al., 2005). Neuroimaging studies have shown that seeing other people's pain activates brain circuitries that partially overlap with those activated by the actual painful experience (Morrison et al., 2004; Singer et al., 2004; Botvinick et al., 2005; Jackson et al., 2005). This automatic link with our own experience suggests that seeing human physical suffering could influence the brain processing (and the subjective experience) of pain stimuli received concomitantly. However, functional magnetic resonance imaging (fMRI) studies have not specifically investigated how we respond to noxious stimuli while empathizing with others.

Behavioral data indicate that seeing unpleasant pictures illustrating disgust, fear, anger, or pain increase subjective pain intensity (Wunsch et al., 2003) and decrease pain tolerance (de Wied and Verbaten, 2001; Meagher et al., 2001). Although images showing physical suffering were included in these studies, their specific effect on pain was never tested. It remains, therefore, unknown whether facing others in pain produces stronger effects

Received May 27, 2006; revised Aug. 30, 2006; accepted Aug. 30, 2006.

This work was supported by grants from the UPSA Foundation, Fondation Mécénat CNP, and Fondation pour la Recherche Médicale. We thank Alex Mertens for his contribution toward the preparation and conduction of the recordings.

Correspondence should be addressed to Fabio Godinho, Institut National de la Santé et de la Recherche Médicale (INSERM), Equipe Mixte INSERM 342, Central Integration of Pain, Hôpital Neurologique Pierre Wertheimer, 59 Boulevard Pinel, 69003 Rez-de-Jardin Lyon, France. E-mail: fabiogodinho@wanadoo.fr.

DOI:10.1523/JNEUROSCI.2260-06.2006

Copyright $\odot 2006$ Society for Neuroscience $\quad$ 0270-6474/06/2611454-08\$15.00/0 on our own somatic sensations than seeing stimuli similarly unpleasant but lacking human pain contents.

The processing level at which emotional states modulate pain is also a matter of controversy. Previous fMRI studies of empathy insisted that low-level sensory networks [primary and second somatosensory areas (SI-SII) and posterior insula] remained silent, and concluded that only cortical regions processing affective qualities of pain underlay empathy-related phenomena (Singer et al., 2004; Botvinick et al., 2005). Therefore, one may hypothesize that any concomitant modulation of pain perception while facing another's pain should also operate through high-order networks. If this were so, pain modulation by empathy should operate through different mechanisms than those of selective attention, because attention changes pain perception by acting, at least in part, on low-level somatic areas (Hofbauer et al., 2001; Bantick et al., 2002). However, others have suggested that negative emotions such as anxiety and fear do enhance selective attention to pain (Arntz et al., 1991; Janssen and Arntz, 1996), which should predict an effect on early sensory responses. These different hypotheses may be tested using spatiotemporal analysis of somatosensory brain evoked-potentials (SEPs), because the attentional effects on pain processing and the response properties of SII/ posterior insula to somatosensory inputs are well characterized in the time domain (Desmedt et al., 1983; Garcia-Larrea et al., 1991; Frot and Mauguiere, 2003).

Herein, we investigated how the subjective intensity of electrical stimuli was modulated when participants viewed pleasant or unpleasant pictures. These were segregated into subsets depicting or not human physical sensations (showing either human pain or somatic pleasure). Thus, we assessed whether pictures supposed to empathically activate the neural representations of somatosensation had a greater ability to modulate pain than images of same 

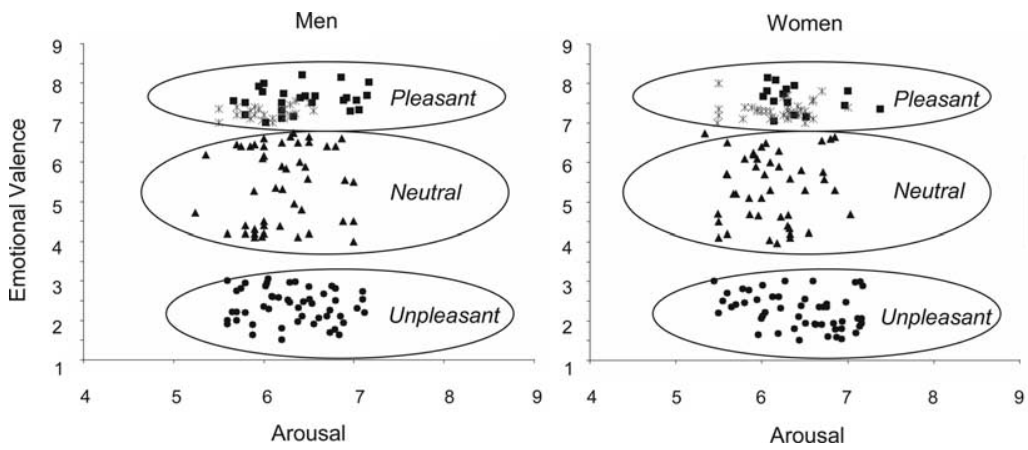

Figure 1. Valence and arousal values of emotional pictures. Valence (1, very unpleasant; 5 , neutral; 9 , very pleasant) and arousal (1, calm; 9 , excited) ratings were plotted for each image in men's and women's paradigms. Values assigned to unpleasant, pleasant, and neutral sets were taken from IAPS data. Additionally, images from several internet sources were included in the pleasant group to strengthen valence of women's pleasant subset and to introduce pleasant pictures explicitly related to human somatic experience. Valence and arousal ratings for these pictures were obtained in a pilot study with 10 men and 10 women. As a result, arousal and valence dimensions were matched, and comparable emotional manipulation was obtained in both genders.

Table 1. Valence and arousal values for each category of pictures

\begin{tabular}{lll}
\hline Picture categories & Emotional valence & Arousal \\
\hline Pleasant-body & $7.40 \pm 0.98$ & $6.10 \pm 1.40$ \\
Pleasant-nonbody & $7.45 \pm 0.85$ & $6.40 \pm 1.10$ \\
Neutral-body & $5.30 \pm 1.20$ & $6.05 \pm 1.04$ \\
Neutral-nonbody & $5.43 \pm 0.95$ & $6.00 \pm 0.90$ \\
Unpleasant-body & $2.10 \pm 0.70$ & $6.45 \pm 0.80$ \\
Unpleasant-nonbody & $2.20 \pm 0.93$ & $6.39 \pm 1.15$ \\
\hline
\end{tabular}

Valence scores range from 1 (very unpleasant) to 9 (very pleasant), whereas arousal scores range from 1 (calm) to 9 (excited). Values did not differ significantly between body and nonbody subsets for both pleasant and unpleasant groups. Values are expressed as mean \pm SD.

emotional valence but lacking somatosensory contents. Behavioral responses were coupled to high-density electroencephalogram (EEG) and source localization techniques to assess the timing and the structures implicated in the emotional modulation of pain.

\section{Materials and Methods}

Subjects. Sixteen healthy, right-handed subjects (eight men; eight women), 21-35 years of age (mean age, 25 years), participated in the experiment. They were recruited by announcement in University Colleges and were not paid for their participation. All subjects were free of neurological and psychiatric symptoms or signs and naive to the experimental procedure. The study was approved by the local Ethics Committee (University of St. Etienne, St. Etienne, France). All subjects provided informed consent and received verbal and written instructions about all details of the experiment and about the possibility to withdraw at any moment.

Visual stimuli. Participants were seated in a dark room, $2 \mathrm{~m}$ in front of the pictures, which were projected onto a white screen. Each image was presented over an area of $60 \times 40 \mathrm{~cm}$ and subtended a visual angle of $5.2^{\circ}$ in the horizontal plane and $3.5^{\circ}$ in the vertical plane. On preliminary experiments, such dimensions were found to be large enough to induce powerful emotional modulation, while allowing the subjects to scan the pictures with minimal eye movements, thus minimizing eye artifacts.

Two-hundred forty images were used in this study, $75 \%$ of which were chosen from the International Affective Picture System (IAPS) (Lang et al., 2001). We selected pictures previously validated as emotionally pleasant (valence, $>7$ ), unpleasant (valence, $<3$ ), or neutral (valence, 4-6.5) (Fig. 1). Aiming to control attentional factors, the range of arousal values of pictures was kept identical across valence subcategories. A tendency for lower arousal levels was observed for neutral pictures compared with either pleasant or unpleasant images, but the trend was not significant. Because the IAPS database did not contain a sufficient number of pic- clearly discernable.

tures with explicit contents related to body sensations, and also because arousal values of pleasant IAPS pictures were lower for women than for men, we included a number of images taken from other sources ( $25 \%$ of the total) presenting explicit somatic contents and providing high arousal in women. These non-IAPS color pictures had the same digital IAPS pictures' dimension $(1020 \times 760$ pixels $)$ and were taken from several advertisements and databases on the internet (images available on demand). Arousal and valence levels associated with these pictures were rated in a pilot experiment by 20 participants (10 males and 10 females) who did not participate in the main paradigm. Using the same scoring methods as described in the original IAPS database, the ratings for non-IAPS images fit well within the valence span of IAPS data. Figure 1 illustrates the valence and arousal value distribution of the full set of images, in which IAPS and non-IAPS-derived points are

Because men and women may show different affective reaction patterns to emotional stimuli (Bradley et al., 2001; Sabatinelli et al., 2004), the set of pictures used in the men's paradigm were partly different from that used in the women's paradigm ( $75 \%$ of different images). This allowed matching both emotional valence $\left(t_{(298)}=1.53\right.$; two-tailed $p=$ $0.13)$ and arousal values $\left(t_{(298)}=0.93\right.$; two-tailed $\left.p=0.35\right)$ between men and women (Fig. 1).

According to their emotional valence, pictures were divided into three main sets of 80 items each, containing the following, respectively: (1) pleasant, (2) unpleasant, and (3) neutral images. Each set of images was further split into two groups as a function of the existence or not of explicit references to somatic sensations (corporality). Hence, six emotional conditions were tested, namely: pleasant-body, pleasant-nonbody, neutral-body, neutral-nonbody, unpleasant-body, and unpleasantnonbody. As shown in Table 1, emotional valence scores were virtually identical in the body and nonbody subsets for both pleasant $\left(t_{(78)}=0.85\right.$; two-tailed $p=0.39)$ and unpleasant $\left(t_{(78)}=0.38\right.$; two-tailed $\left.p=0.71\right)$ pictures. Similarly, a lack of significant difference was also noted for arousal values between the body and nonbody groups for both pleasant $\left(t_{(78)}=1.34\right.$; two-tailed $\left.p=0.19\right)$ and unpleasant images $\left(t_{(78)}=1.27\right.$; two-tailed $p=0.21$ ).

The unpleasant-body category was composed of 40 images showing explicitly acute pain in human beings, such as burnings, amputations, wounds, and the like. The unpleasant-nonbody subset comprised scenes of violence, guns, suicide tentative, accidents, and disgust. The pleasantbody category showed humans feeling pleasure from having their bodies being caressed by massages or waterfalls, as well as enjoying jacuzzis, vapor baths, saunas, etc. To encourage the empathy with the feelings depicted in the body scenes, we used pictures showing men in the men's paradigm and pictures showing women in the women's paradigm.

The pleasant-nonbody images were those that differed mostly between the men's and women's paradigms. Those in the women's paradigm exhibited fashionable clothes, lingerie, perfumes, and physically attractive men in artistic poses. For men's paradigm, scenes involved sports, cars, motorcycles, partially undressed women, etc. We avoided explicit sexual scenes because these images were rated as much more arousing and pleasant by men than by women, and might have biased the results (de Wied and Verbaten, 2001). The neutral subset included complex visual stimuli with no particular emotional content for the average population, such as parachute falling, snow skiing or waterskiing, natural phenomena (volcano, lavas, cyclone), and animals. Each picture was repeated two times along the experiment, but never in the same block. To minimize the effects of repetition, pictures were modified whenever repeated (by turning them left/right, or detailing a particular part of the scene).

Electrical stimuli. Square pulses of $500 \mu$ s duration were delivered to the second and third digits of the hand through ring electrodes. Left and 


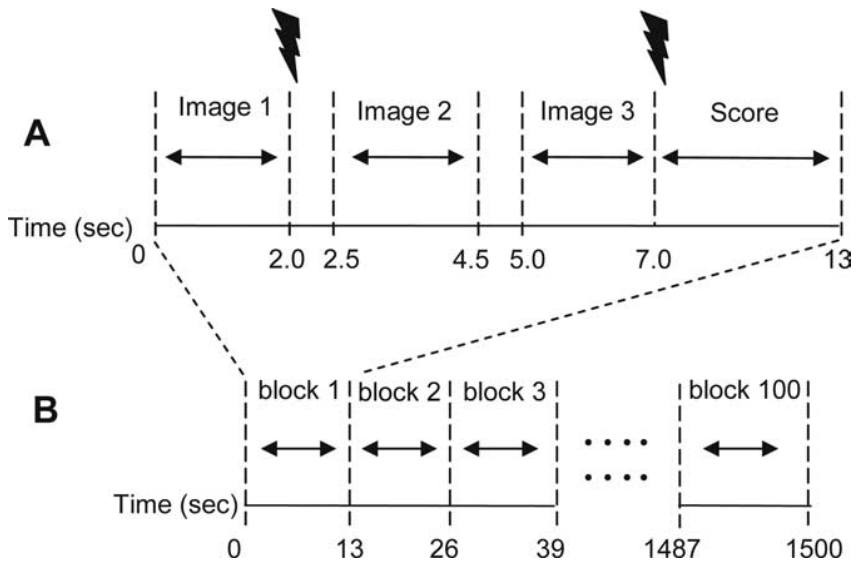

Figure 2. Structure of the block design paradigm. $\boldsymbol{A}$, Internal structure of one block. Three images with same emotional valence and two electrical stimuli of same intensity composed each block of 13 s duration. Electrical stimuli were delivered after the first and the third pictures. $\boldsymbol{B}$, The whole paradigm had a sequence of 100 blocks (25 min time length).

right sides were stimulated in an equal number of subjects. This procedure was aimed to avoid unilateral brain activation effects predominant in the hemisphere contralateral to stimulation, which could have biased the electrophysiological source analysis. Electrical shocks were delivered after the first and the third images of each block, as illustrated in Figure 2. Two stimulus intensities (painful and innocuous) were used. Both intensities were determined individually using the method of limits. The lower intensity $(10-15 \mathrm{~mA})$ was set inferior to the participant's pain threshold, and was quoted $1-3$ on a numerical rating scale (NRS), in which $0=$ no sensation, $4=$ pain threshold, and $10=$ unbearable pain. The higher intensity (25-30 mA) was set slightly superior to pain threshold (NRS, 5-6). Participants were informed that several stimulus intensities would be used during the experiment, whose values could vary randomly between the lower and higher intensities previously determined. They were, therefore, not aware that only two fixed intensities were to be used. A total number of 200 stimuli (100 painful/100 innocuous) were delivered in each experiment.

Apparatus and recordings. The EEG was continuously recorded using a 64-electrode cap (Eletrocap), with all electrodes referred to the nose. The electro-oculogram was monitored by a tin electrode attached to the inferior eyelid and also referred to the nose. A ground electrode was placed on the forehead. Electrode/skin impedances were kept $<2 \mathrm{k} \Omega$. Somatosensory responses were amplified by a 12 bit digitizer (vertical resolution of $0.1 \mu \mathrm{V} / \mathrm{bit}$ ), with a bandpass of $0.3-70 \mathrm{~Hz}$ (Ceegraph XL; Bio-logic Systems, Mundelein, IL). Signals were sampled at $256 \mathrm{~Hz}$ and epoched over an analysis time of $1097 \mathrm{~ms}$, which included $97 \mathrm{~ms}$ of prestimulus delay used for baseline computation. An automatic artifact rejection system excluded from the average all trials containing transients exceeding $\pm 65 \mu \mathrm{V}$ at any recording channel, including the electro-oculogram. Furthermore, the quality of SEP traces was ensured by careful visual inspection in every subject and trial, excluding all epochs containing blink artifacts and applying appropriate digital, non-phase shift low-pass filters at $30 \mathrm{~Hz}$. SEP traces in Figure 5 show that even the most lateral electrodes were not significantly contaminated with muscle artifacts. The paradigm was edited and run by the Visual Task Editor and the Visual Stimulator from Orgil Medical Equipments (Ayala, Israel). Each event in the paradigm (visual or electrical) was associated with a digital code (trigger) that was sent to the continuous EEG, allowing off-line segmentation and average of selected EEG periods for analysis. The electrical stimulator was triggered by the visual stimulator, thus allowing a very accurate synchronization between visual and electric stimuli. SEPs were processed and analyzed by Brain Performance Measurement (BPM) system software (Orgil Medical Equipments) and by Neuroscan (Compumedics, Hamburg, Germany).

Electrophysiological source analysis. Brain generators associated with emotional modulation of SEPs were estimated by Low Resolution Brain Electromagnetic Tomographic Analysis (LORETA) and Brain Electrical
Source Analysis (BESA 2000; Megis Software, Gräfelfing, Germany). The first method provides an approximate three-dimensional solution of the inverse EEG problem, aiming to determine the most active brain regions in a given instant of time (Pascual-Marqui et al., 1994). Compared with usual dipole-based methods, LORETA does not need a priori hypotheses regarding field distribution of active sources. Brain areas were regarded as active when the activation value exceeded by at least $3 \mathrm{SD}$ of the mean prestimulus baseline. This analysis was first applied to the average painful SEPs to identify the most active areas for each of the six emotional conditions. For this, the time analysis was divided in four periods of analysis: $t_{1}, 50-150 \mathrm{~ms} ; t_{2}, 180-270 \mathrm{~ms} ; t_{3}, 270-400 \mathrm{~ms}$; and $t_{4}, 400-500 \mathrm{~ms}$. These time periods were chosen from Global Field Power peaks of grand SEP averages (Lehman and Skrandies, 1980). Global Field Power estimates spatial variance for each sample point, and provides a "neutral" approach allowing us to determine the periods of time during which such variance is maximal (i.e., when focal activities are present). Bilateral (but not symmetrical) activation was observed in all conditions as following: at 50-150 ms, inferior parietal lobule, medial and lateral prefrontal areas, and middle temporal gyrus; at $180-270 \mathrm{~ms}$, active areas were superior and inferior parietal lobule, post-central gyrus, and precuneus; at $270-$ $400 \mathrm{~ms}$, active areas were inferior parietal lobule, precuneus, post-central gyrus, and prefrontal areas; and last, at $400-500 \mathrm{~ms}$, the most active areas were middle temporal gyrus, prefrontal areas, and precuneus. Finally, we applied LORETA to the subtractions of SEP traces between those emotional conditions yielding significant SEP amplitude differences (see Fig. 5 ). These same subtractions were further modeled by spatiotemporal dipoles (BESA 2000) (Scherg, 1990), fitted over the time interval showing significant differences of SEP amplitudes. Dipole coordinates were then projected onto the standardized space of Talairach and Tournoux (Talairach et al., 1957) and integrated with LORETA results.

Experimental paradigm. From the outset, participants were told they would define by themselves the intensity of the most painful stimulus they wanted to tolerate (based on their pain threshold) and that no stimulus in the experiment would exceed this intensity. The nociceptive character of suprathreshold stimuli was ascertained by simultaneous recording the nociceptive-flexion reflex. Afterward, a test paradigm comprising 24 pictures and 16 electric stimuli was run to acquaint the subjects with the overall experiment's structure.

The duration of the whole experiment (excluding electrode preparation and practice trial) was $25 \mathrm{~min}$ and was run in two parts. The paradigm had a "block" design containing 100 blocks, each comprising three pictures and two electrical stimuli (Fig. 2). All pictures in the same block were presented for $2 \mathrm{~s}$, belonged to the same emotional valence subset, and contained closely related thematic contents (e.g., three massage scenes, three burn scenes, and so on). The two electric stimuli of a same block had the same intensity. Subjects rated the average intensity of both shocks at the end of each block (i.e., $\sim 8 \mathrm{~s}$ after the first and $3 \mathrm{~s}$ after the second electric stimulus) using an NRS projected onto the same screen during $6 \mathrm{~s}$. Scores were provided verbally, and values were fed into the EEG recordings by one of the experimenters.

Statistical analysis. The effect of the emotional contents of the images on the subjective intensity of pain was assessed using repeated-measures ANOVA with three within factors: (1) stimulus intensity (painful vs innocuous), (2) emotional valence of the pictures (pleasant vs unpleasant vs neutral), and (3) corporality of the pictures (body-related vs nonbodyrelated). The dependent variable was the subjective pain rating (NRS scores).

The correspondence between behavioral and electrophysiological data were then assessed by comparing SEP amplitudes to painful stimuli between pleasant and unpleasant conditions. SEPs were segmented into four time periods, labeled $t_{1}$ to $t_{4}$ as previously described. On these premises, a repeated-measures ANOVA was applied with three within factors: (1) emotional valence (pleasant vs unpleasant), (2) corporality (body-related vs nonbody-related), and (3) period of analysis $\left(t_{1}\right.$ to $\left.t_{4}\right)$. The dependent value was the mean SEP amplitude from all electrodes (mean value of SEP recorded from 64 electrodes). Degrees of freedom were corrected using the G-G (Greenhouse-Geisser) $\varepsilon$ for correlated measures. Contrast testing between conditions or groups of conditions was conducted only when preceded by significant main factors effects. 

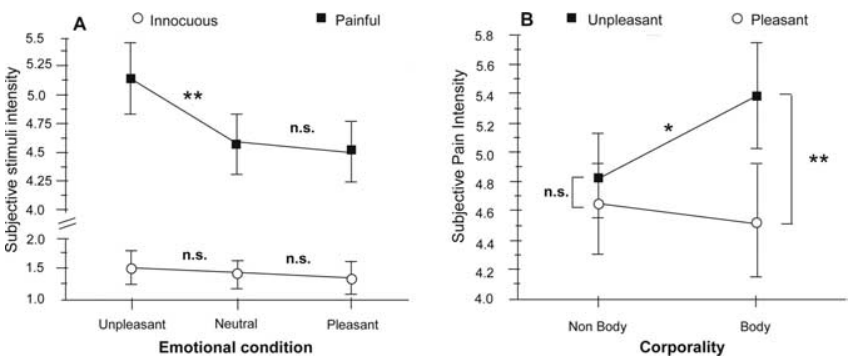

Figure 3. Effects of emotional context on subjective stimulus intensity. $\boldsymbol{A}$, Significant emotional modulation of perceived intensity was only observed for painful stimuli (black squares), but not for innocuous stimuli (white circles) whose perception remained stable despite changing the emotional context. Note also that only unpleasant pictures modulated pain, whereas pleasant pictures did not change significantly the perception of stimuli relative to neutral images. $B$, ANOVA interaction plot shows that the subjective pain ratings were significantly different between pleasant and unpleasant conditions containing explicit references to human body sensations (body). Differences were not significant between pleasant and unpleasant pictures not depicting body sensations (nonbody). Moreover, pain scores were significantly higher during the unpleasant-body than the unpleasant-nonbody condition. Values are expressed as mean $\pm \mathrm{SE}\left({ }^{* *} p<0.01 ;{ }^{*} p<0.05\right)$. Values for neutral pictures were never significant and are not illustrated for graph clarity.

Finally, regression analysis was performed to assess whether the extent of subjective pain modulation correlated with activity changes in brain regions tagged by source analysis. To this aim, significant changes in SEP amplitudes were used as explanatory variable of subjective modulation of pain across the different conditions.

\section{Results}

\section{Behavioral analysis: the effect of emotional conditions on pain ratings}

ANOVA on subjective ratings to electric shocks showed a strong effect of the emotional context where stimuli were delivered $\left(F_{(2,15)}=9.81 ; p=0.0008\right)$. This effect was contingent on stimulus intensity and was only present for painful stimuli [significant interaction between stimulus intensity and emotional valence $\left.\left(F_{(2,30)}=10.60 ; p=0.0005\right)\right]$ (Fig. $\left.3 A\right)$. Contrast analysis showed indeed that pain perception was significantly enhanced during presentation of unpleasant pictures, relative to neutral images $\left(F_{(1,15)}=30.91 ; p=0.0001\right)$. Conversely, no significant difference in pain perception was observed between the pleasant and neutral conditions $\left(F_{(1,15)}=0.39 ; \mathrm{NS}\right)$.

The effect of emotional context on pain perception was also dependent on whether or not the pictures explicitly displayed body-related contents, either pleasant (massages, caresses) or unpleasant (human physical pain). This was reflected by a significant interaction between pictures' corporality and emotional context $\left(F_{(2,30)}=6.99 ; p=0.005\right)$. Post hoc tests showed that differences in pain ratings were highly significant between pleasant-body and unpleasant-body conditions $\left(F_{(1,15)}=37.80\right.$; $p=0.0001)$, as well as between unpleasant-nonbody and unpleasant-body conditions $\left(F_{(1,15)}=8.09 ; p=0.02\right)$. In each case, higher intensity reports were observed in the unpleasant body-related condition. No significant differences in pain ratings appeared when comparing unpleasant and pleasant conditions without somatic contents $\left(F_{(1,15)}=3.04\right.$; ns) (Fig. $\left.3 B\right)$.

To investigate possible specific gender-linked effects, we compared pain ratings between men and women under comparable emotional manipulations. Our data did not reveal any significant difference between genders in neutral $\left(t_{(14)}=0.39\right.$; two-tailed $p=0.70)$; pleasant $\left(t_{(14)}=1.50\right.$; two-tailed $\left.p=0.16\right)$ or unpleasant conditions $\left(t_{(14)}=1.25\right.$; two-tailed $\left.p=0.23\right)$.

Last, no time-dependent linear trend was noted on pain rat-
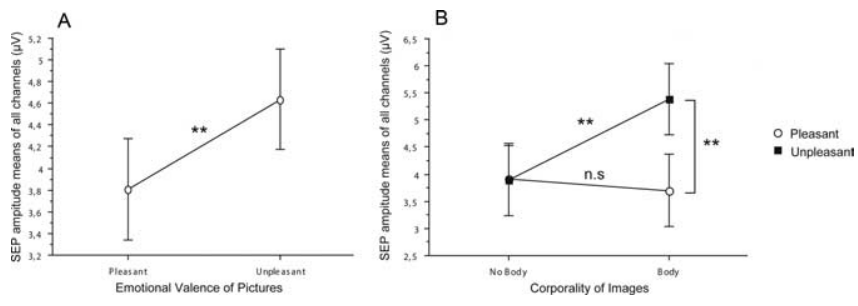

Figure 4. Effects of emotional context on cortical responses to painful stimuli. $A$, Main effect of the emotional condition on cortical responses to painful stimuli. SEP amplitudes were significantly higher during the unpleasant than during the pleasant condition. $\boldsymbol{B}$, ANOVA interaction plot shows that cortical SEP were significantly increased when unpleasant-body pictures were displayed relative to either pleasant-body or unpleasant-nonbody. No significant differences were observed when comparing unpleasant and pleasant conditions without human somatic contents (nonbody). Note that this emotional effect is symmetrical to that described on behavioral pain ratings. Values are expressed as mean $\pm S E\left({ }^{* *} p<0.01\right)$.

ings thorough the experiment, neither in the sense of habituation nor in the sense of sensitization (linear correlation, $r^{2}=0.08, p=$ $0.15)$, probably because of the fact that blocks containing painful stimuli were intermingled with those containing innocuous stimuli (see Materials and Methods, Electrical stimuli).

\section{Electrophysiological analysis: emotional modulation of SEP amplitudes}

We used a three-way ANOVA to test simultaneously the effect of emotional valence (pleasant vs unpleasant), the corporality of pictures (body vs nonbody-related) and the time analysis of SEP (four time windows) on cortical SEP amplitudes (Fig. 4).

The results were consistent with those observed on behavioral analysis, showing that SEP amplitudes to painful stimuli were significantly modulated by the emotional valence of the pictures presented concomitantly $\left(F_{(1,15)}=21.93 ; p=0.0003\right)$ (Fig. $4 A$ ). This effect was dependent on both corporality and time analysis of SEP, as revealed by the significant interaction between valence and corporality $\left(F_{(1,15)}=6.95 ; p=0.02\right)$ and valence and time $\left(F_{(1,15)}=4.01 ; p=0.02\right)$. Similar to behavioral data, post hoc contrasts showed significant differences of SEP amplitudes between unpleasant-body and pleasant-body conditions $\left(F_{(1,15)}=\right.$ $13,60 ; p=0.002)$, but not between pleasant and unpleasantnonbody conditions. Additionally, significant differences were observed between unpleasant-body and unpleasant-nonbody conditions $\left(F_{(1,15)}=10.80 ; p=0.005\right)$, indicating that SEP changes were specifically contingent to the presentation of unpleasant, body-related pictures (Figs. 4B, 5). Concerning the time analysis of SEP, significant differences of amplitudes between emotional valences were observed only for the $270-400 \mathrm{~ms}\left(t_{3}\right)$ $\left(F_{(1,15)}=7.92 ; p=0.01\right)$ and the $400-500 \mathrm{~ms}$ windows $\left(t_{4}\right)$ $\left(F_{(1,15)}=31.48 ; p=0.0001\right)$ as graphically illustrated on Figure 5 .

\section{Electrophysiological source analysis}

The period of analysis when SEPs demonstrated the earliest changes associated to differential pain reports $(270-400 \mathrm{~ms})$ underwent source analysis to determine the cortical regions differentially activated. To this aim, the subtraction of SEP traces between the unpleasant-body and pleasant-body, as well as the subtraction between the unpleasant-body and unpleasantnonbody conditions were assessed using LORETA and BESA 2000. LORETA provides an approximate three-dimensional solution to the inverse EEG problem and estimates the most active cortical regions within a given time interval (see Materials and Methods). This method identified a small set of regions whose activity differed significantly between emotional conditions for 

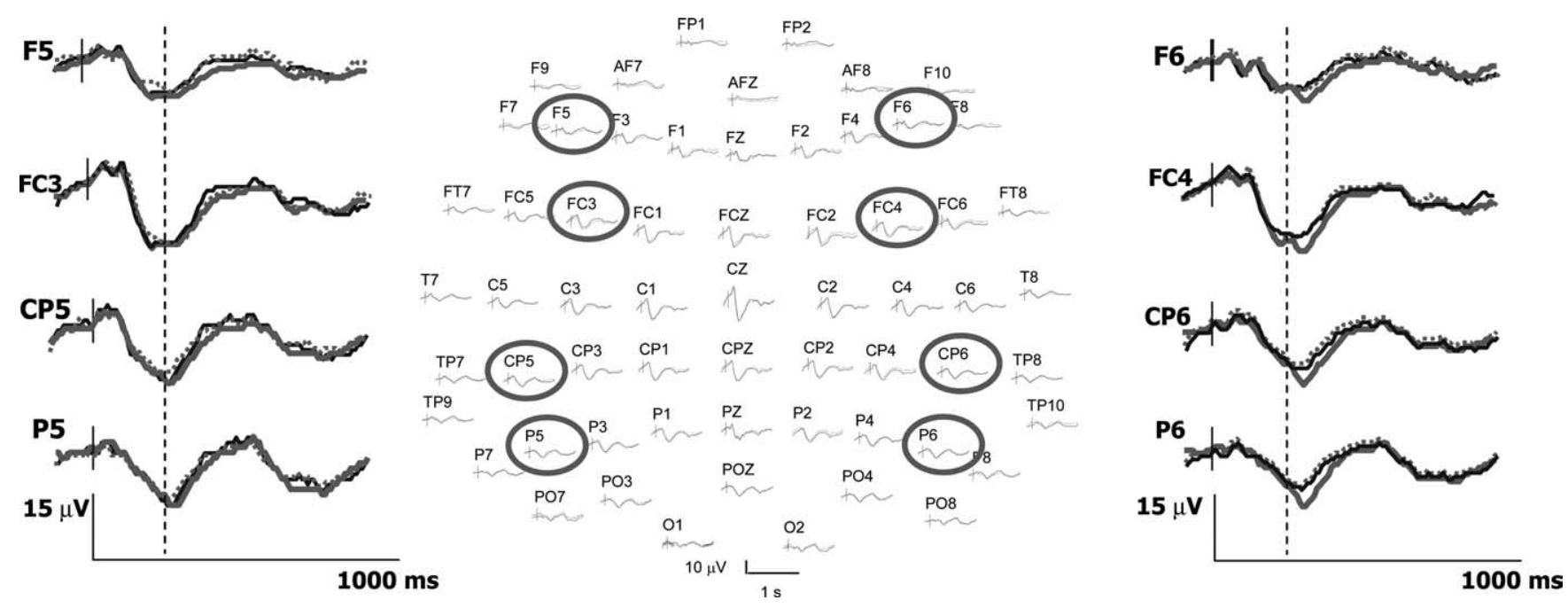

Figure 5. Cortical responses to painful stimuli associated or not to emotional modulation of pain. The 64-channel SEPs at their scalp positions (nose up) are represented in the center. Positive voltages are represented downward. Traces from eight selected electrodes (circles) are enlarged in the left and in the right parts to illustrate that SEP amplitudes to painful stimuli were significantly enhanced during the unpleasant-body condition (gray traces) relative to both unpleasant-nonbody (dotted) and pleasant-body (black) conditions. Note also that amplitude differences occurred only after 270 ms poststimulus (dotted line) and concerned exclusively the electrodes located on the right side of the scalp. See Results for details.

which pain reports also differed. These regions were lateralized toward the right hemisphere whatever the side electrically stimulated and comprised the dorsolateral prefrontal cortex (DLPFC), the temporooccipital cortex, and the temporal pole (Fig. 6). Of notice, the left DLPFC and temporal cortices showed some differential activity too, although they did not reach statistical significance (see Materials and Methods). The same regions were observed for both SEP subtractions (i.e., unpleasant-body minus pleasant-body and unpleasant-body minus unpleasant nonbody). Additional analysis at the subsequent time window (400-500 ms) showed a larger pool of areas differentially activated, including the bilateral DLPFC, the right temporal pole, and bilateral superior parietal areas.

An additional analysis was performed over the 270-400 ms epoch using dipolar sources. A satisfactory solution [mean residual variance (RV), 9.15\%; best RV, $6.00 \%$ ] was obtained with sources located on right $(X \mathrm{t}=49.4, Y \mathrm{t}=20.9, \mathrm{Zt}=60)$, and left prefrontal areas $(X \mathrm{t}=-46.3, Y \mathrm{t}=$ $1.2, Z \mathrm{t}=16.9)$, right posterior parietal $(X \mathrm{t}=55.9, \mathrm{Yt}=-68.6, \mathrm{Zt}=40.6)$, right occipito-temporal $(X \mathrm{t}=66.6, \mathrm{Yt}=-74.6$, $Z \mathrm{t}=9)$, and right temporal pole $(X \mathrm{t}=50.9, \mathrm{Yt}=15.5, Z \mathrm{t}=$ -8.7). This solution was concordant with that obtained by LORETA, as shown in Figure 6. In no instance dipolar sources went spontaneously to somatosensory regions. Forcing new sources in SI and/or SII did not yield any significant gain of the solution $(<0.5-1 \% \mathrm{RV}$ reduction). We therefore concluded that, during the time window showing condition-related SEP amplitude changes, differential brain activation did not concern SI-SII somatosensory regions.

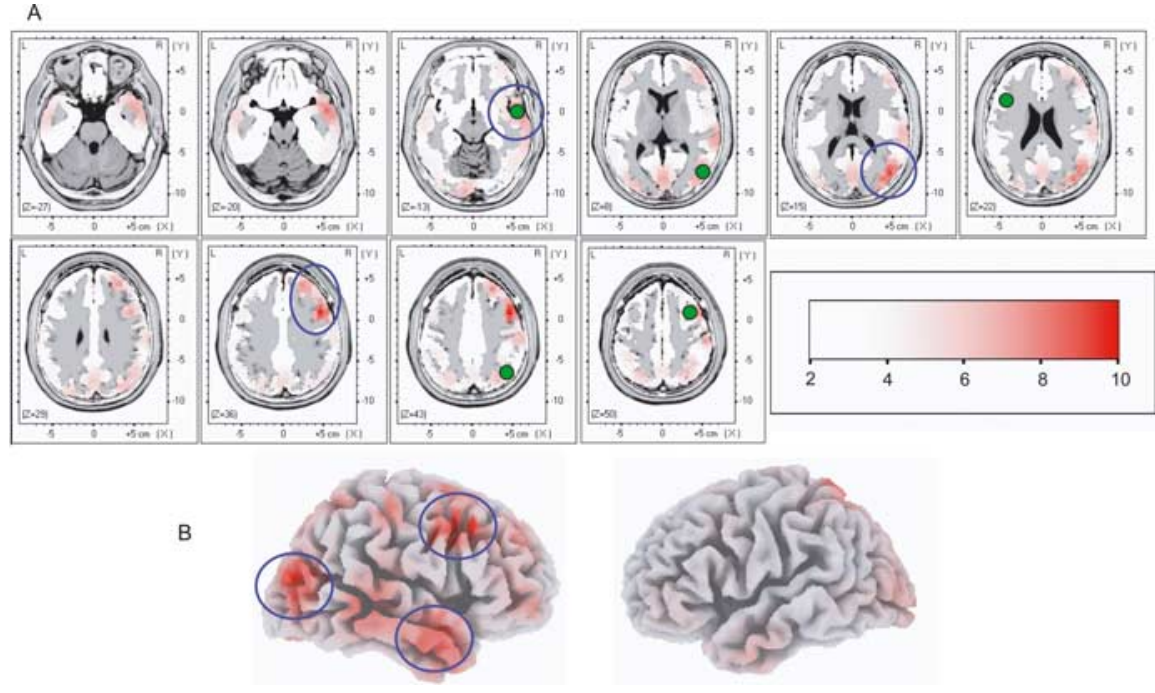

Figure 6. Anatomical (LORETA and BESA) modeling of cortical regions showing the earliest activity associated with emotional modulation of pain. $A$, The difference (subtraction) between SEP traces obtained during unpleasant- and pleasant-body conditions, as well as between unpleasant-body and unpleasant-nonbody conditions, were assessed by LORETA within $270-400 \mathrm{~ms}$, when significant differences of SEP amplitudes were observed. Cortical areas of maximal activity are illustrated on normalized Talairach slices. These areas were lateralized toward the right hemisphere and concerned the DLPFC, the temporal pole, and the temporo-occipital junction (blue circles). Only regions whose differential activities exceeded by at least 3 SD of the mean prestimulus baseline are illustrated. The Z-levels in the color scale correspond to the number of SDs from the mean prestimulus baseline. Additionally, dipole analysis was performed on the same epoch (green circles). Dipolar sources lay close to the most active areas showed by LORETA. No sources were found over SI/SII or posterior insula with either technique. $\boldsymbol{B}$, Three-dimensional representation of both hemispheres showing the cortical regions aforementioned (blue circles).

To assess whether subjective pain modulation was correlated with brain responses, multiple regression analysis was performed using SEP amplitude ratios between pleasant and unpleasant body conditions in the three most significant regions as explanatory variables of the subjective increase of pain reports. A significant linear relationship $\left(R^{2}=0.72 ; p=0.029\right)$ was indeed found among the variables, modeled by the following formula: $[A:]=$ $1.103+0.08021 \times[B:]+0.006913 \times[C:]+0.03849 \times[D:]$, where $A$ was subjective pain increase, and $B, C$, and $D$ were the activities at prefrontal, temporal pole, and temporo-occipital re- 
gions, respectively. The multiple regression function passed nearly through the origin of coordinates, suggesting that the absence of SEP changes corresponded to the lack of increased pain reports. No single region correlated significantly with pain reports, only their combination, in accordance with the notion that a combination of activities in the three regions may have underlay the difference of pain reports between unpleasant and pleasantbody conditions.

\section{Discussion}

\section{Behavioral responses: the role of empathy on pain perception}

The subjective experience of acute pain was increased by the concomitant presentation of scenes showing human physical pain. Conversely, unpleasant pictures of same emotional levels, but without explicit references to physical pain evoked no significant effects on pain perception. This could not be explained by differences in picture-related arousal, the values of which were matched across the categories.

A general effect of aversive pictures on behavioral pain responses was shown in previous studies (de Wied and Verbaten, 2001; Meagher et al., 2001; Wunsch et al., 2003). Although human pain scenes were included in these, their specific effect was never assessed. Our findings are novel because they illustrate that only the confrontation with images explicitly depicting physical pain modulated the somatic perception. Thus, the specific representation of human physical suffering is a powerful factor of pain modulation, which we suggest may act through an automatic reaction of empathy. This hypothesis is supported by studies showing that observation of other people's pain activates our own inner pain representations (Morrison et al., 2004; Singer et al., 2004; Jackson et al., 2005). According to our results, such empathy-induced activation of pain circuitries may also transiently amplify our own pain perception.

It is noteworthy that pleasant body-related images failed to modify significantly pain ratings. We included very few explicit sexual materials within pleasant images to minimize attentional effects contamination (de Wied and Verbaten, 2001; Lang et al., 2001; Rhudy and Williams, 2005). Although this may have affected the inability of pleasant pictures to modulate pain, by making them deviate less from neutral data than unpleasant images (Fig. 1), such inability was also found in previous studies that did not eliminate sexual material (Wunsch et al., 2003). Human conditioning theories state that the transfer of affective reactions from an unconditioned to a conditioned stimulus is facilitated when both belong to the same affective category (Cook et al., 1986; Hamm et al., 1989; Ohman and Mineka, 2001). Our results support this notion by showing that the facilitatory effect of aversive images on painful stimuli is greater than the possible inhibitory effect of appetitive images on pain.

Few studies have suggested that possible differences in emotion and arousal between men and women may differentially alter pain processing (Rhudy et al., 2005). In our experiment, both valence and arousal levels triggered by pictures were made equivalent across genders by using different sets of pictures. On these conditions, no significant gender differences in pain ratings were observed, suggesting that, when emotional manipulation is adequately matched, its effect on pain may be similar in men and women.

\section{Time analysis of cortical pain-related responses: emotion versus attention}

Electrophysiological studies have demonstrated that painful electrical stimuli activate SI, SII, and posterior insula in the $20-150$ ms range (Valeriani et al., 2000; Frot et al., 2001; Barba and Valeriani, 2004; Stancak et al., 2005). Conversely, our data showed that earliest cortical changes associated with increased pain occurred later than $270 \mathrm{~ms}$, suggesting that pain processing at lowlevel sensory structures was not affected by emotional states. Source analysis pointed to high-level structures at the right hemisphere as covariates of emotion-induced changes in pain perception: the right DLPFC, the right temporal pole, and the right temporo-occipital junction were the earliest and most prominent regions associated to higher pain ratings.

Numerous experiments by our group and others have shown that attention modifies cortical SEPs at very early latencies, commonly between 40 and 150 ms poststimulus (Desmedt and Robertson, 1977; Desmedt et al., 1983; Michie et al., 1987; Desmedt and Tomberg, 1989; Garcia-Larrea et al., 1991, 1995; Mauguière et al., 1997). This is supposed to index the action of top-down mechanisms over low-level sensory structures, and is in agreement with neuroimaging and intracranial EEG studies demonstrating early activity changes in SI, SII, insula, and anterior cingulate cortex (Hofbauer et al., 2001; Bantick et al., 2002; Ohara et al., 2004). In contrast with this, emotional effects on cortical SEPs were in our experiment much later than attentional effects previously reported. The absence of similar early findings herein cannot be attributed to technical factors or lack of SEP sensitivity. Rather, our results support the notion that pain modulation induced by scenes of human physical suffering did not implicate attention as the predominant factor. This hypothesis does not imply that attentional mechanisms would not apply in everyday conditions, where attention and emotion probably interact. However, it underlines that emotion and selective attention may both modulate pain perception and cortical responses through distinct mechanisms, which can be separated by appropriate experimental settings.

\section{Mechanisms of emotion-driven pain modulation}

Among the structures significantly associated to pain modulation, those with the earliest and most significant participation were found in the right hemisphere, including part of the DLPFC, the temporal pole, and the temporo-occipital junction. These are associative regions involved in high-order cognitive, emotional, and memory processes. The temporo-occipital junction belongs to the ventral stream of visual processing, acting as a link between visual association and memory. Whereas in the left hemisphere this region participates in word encoding (Heun et al., 2000; Galaburda and Cestnick, 2003), in the right hemisphere it seems to be engaged in the assessment of emotional stimuli (Geday et al., 2003; Mesad et al., 2003; Mobbs et al., 2003). The right temporal pole has been implicated in the encoding of emotional stimulus, such as those triggered by fear faces (Kimbrell et al., 1999) or pain-specific anxiety (Masaoka and Homma, 2000). Last, the DLPFC is a crucial component of working memory (WM), a network that allows a mnemonic trace to be temporally stored until used to produce a response (Fuster, 1973; Kojima and Goldman-Rakic, 1982). WM processes in DLPFC are crucial for the subjective continuity of thought, which they support by providing an interface between perception, long-term memory, and action (Levy and Goldman-Rakic, 2000; Baddeley, 2003; Passingham and Sakai, 2004). Previous studies have provided evidence that the role of DLPFC in WM needs coordination with posterior regions, notably parietal and occipitotemporal cortices (Miller and Cohen, 2001; Wood and Grafman, 2003). Its connections with the ventromedial prefrontal cortex, the temporal pole, the anterior insula, and the amygdala (Miller and Cohen, 2001; 
Kondo et al., 2005) support its role on the integration of emotional information and memory. Interestingly, interaction between DLPFC and temporal pole may have important role in imparting affective tone to short-term memories, because there is strong evidence of right temporal pole involvement in both encoding and retrieval of emotional events (Kimbrell et al., 1999; Dolan et al., 2000; Persson and Nyberg, 2000).

The finding that systems involved in detection, analysis, and encoding of negatively biased emotional stimuli were activated along with WM areas supports the hypothesis that the emotional context in which painful stimuli were delivered influenced their encoding in WM buffers. Working memory processes were essential to our paradigm, because subjects did not score pain immediately, but $4-8 \mathrm{~s}$ after receiving the stimulus. We therefore argue that the short-term memory encoding of stimulus intensity during this period may have been biased toward higher levels when painful stimuli were delivered together with images illustrating human pain. This implies that the storage in WM would not be a "neutral" and faithful representation of the actual stimulus characteristics, but rather would depend on the context in which stimuli are delivered.

Similar joint activations of temporo-occipital, temporal pole, and lateral frontal cortices but in the left hemisphere were described during funny cartoon identification (Mobbs et al., 2003). The finding that similar networks can be recruited in opposite hemispheres by appetitive and aversive stimuli is interesting and agrees with the relative predominance of left- and right-sided networks for, respectively, approach and withdrawal-related behaviors (Kimura et al., 2004; Demaree et al., 2005).

Our results did not disclose differential activation of medial temporal structures and amygdala, commonly implicated on emotional processing of affective pictures. This may be attributable to the fact that the responses we recorded were time-locked to painful stimuli, reflecting somatic rather than visual processing. Moreover, there is compelling evidence that the beginning of second-stage declarative memory formation in mesial temporal structures is quite late ( $\sim 500 \mathrm{~ms}$ ) (Fernandez et al., 1999) and may have been missed in our analysis.

\section{General conclusions and summary}

Emotionally laden images representing human pain had a unique capacity to enhance pain reports. Such ability was not shared by images with identical levels of unpleasantness but without somatic contents. This effect was associated with activity changes in the right hemisphere occurring relatively late $(>250 \mathrm{~ms})$ and did not involve low-level somatosensory but rather associative cortices, including temporo-occipital junction, temporal pole, and DLPFC. We suggest that higher pain scores while seeing other people's pain reflected a bias in the way such stimuli were encoded in WM buffers. Therefore, empathic emotions could change the way stimuli are later remembered by acting directly on their memory encoding, without necessarily changing their sensory processing. This is fundamentally different from selective attentional mechanisms of pain modulation.

\section{References}

Arntz A, Dreessen L, Merckelbach H (1991) Attention, not anxiety, influences pain. Behav Res Ther 29:41-50.

Baddeley A (2003) Working memory: looking back and looking forward. Nat Rev Neurosci 4:829-839.

Bantick SJ, Wise RG, Ploghaus A, Clare S, Smith SM, Tracey I (2002) Imaging how attention modulates pain in humans using functional MRI. Brain 125:310-319.

Barba C, Valeriani M (2004) Assessing somatosensory evoked potential
(SEP) generators by human intracranial recordings. Clin Neurophysiol 115:488-489.

Botvinick M, Jha AP, Bylsma LM, Fabian SA, Solomon PE, Prkachin KM (2005) Viewing facial expressions of pain engages cortical areas involved in the direct experience of pain. NeuroImage 25:312-319.

Bradley MM, Codispoti M, Sabatinelli D, Lang PJ (2001) Emotion and motivation II: sex differences in picture processing. Emotion 1:300-319.

Cook III EW, Hodes RL, Lang PJ (1986) Preparedness and phobia: effects of stimulus content on human visceral conditioning. J Abnorm Psychol 95:95-207.

Demaree HA, Everhart DE, Youngstrom EA, Harrison DW (2005) Brain lateralization of emotional processing: historical roots and a future incorporating “dominance.” Behav Cogn Neurosci Rev 4:3-20.

Desmedt JE, Robertson D (1977) Differential enhancement of early and late components of the cerebral somatosensory evoked potentials during forced-paced cognitive tasks in man. J Physiol (Lond) 271:761-782.

Desmedt JE, Tomberg C (1989) Mapping early somatosensory evoked potentials in selective attention: critical evaluation of control conditions used for titrating by difference the cognitive P30, P40, P100 and N140. Electroencephalogr Clin Neurophysiol 74:321-346.

Desmedt JE, Huy NT, Bourguet M (1983) The cognitive P40, N60 and P100 components of somatosensory evoked potentials and the earliest electrical signs of sensory processing in man. Electroencephalogr Clin Neurophysiol 56:272-282.

de Wied M, Verbaten MN (2001) Affective pictures processing, attention, and pain tolerance. Pain 90:163-172.

Dolan RJ, Lane R, Chua P, Fletcher P (2000) Dissociable temporal lobe activations during emotional episodic memory retrieval. NeuroImage 11:203-209.

Fernandez G, Effern A, Grunwald T, Pezer N, Lehnertz K, Dumpelmann M, Van Roost D, Elger CE (1999) Real-time tracking of memory formation in the human rhinal cortex and hippocampus. Science 285:1582-1585.

Frot M, Mauguiere F (2003) Dual representation of pain in the operculoinsular cortex in humans. Brain 126:438-450.

Frot M, Garcia-Larrea L, Guenot M, Mauguiere F (2001) Responses of the supra-sylvian (SII) cortex in humans to painful and innocuous stimuli. A study using intra-cerebral recordings. Pain 94:65-73.

Fuster JM (1973) Unit activity in prefrontal cortex during delayed-response performance: neuronal correlates of transient memory. J Neurophysiol 36:61-78.

Galaburda AM, Cestnick L (2003) Developmental dyslexia. Rev Neurol 36:S3-S9.

Garcia-Larrea L, Bastuji H, Mauguiere F (1991) Mapping study of selective attentional effects on somatosensory evoked potentials. Electroencephal Clin Neurophysiol 82:101-114.

Garcia-Larrea L, Lukaszewicz AC, Mauguiere F (1995) Effects of spatial attention on the somatosensory N1: the N120-to-N140 transition. Psychophysiology 32:526-537.

Geday J, Gjedde A, Boldsen AS, Kupers R (2003) Emotional valence modulates activity in the posterior fusiform gyrus and inferior medial prefrontal cortex in social perception. NeuroImage 18:675-684.

Goubert L, Craig KD, Vervoort T, Morley S, Sullivan MJ, de C Williams AC, Cano A, Crombez G (2005) Facing others in pain: the effects of empathy. Pain 118:285-288.

Hamm AO, Vaitl D, Lang PJ (1989) Fear conditioning, meaning, and belongingness: a selective association analysis. J Abnorm Psychol 98:395-406.

Heun R, Jessen F, Klose U, Erb M, Granath D, Freymann N, Grodd W (2000) Interindividual variation of cerebral activation during encoding and retrieval of words. Eur Psychiatry 15:470-479.

Hofbauer RK, Rainville P, Duncan GH, Bushnell MC (2001) Cortical representation of the sensory dimension of pain. J Neurophysiol 86:402-411.

Jackson PL, Meltzoff AN, Decety J (2005) How do we perceive the pain of others? A window into the neural processes involved in empathy. NeuroImage 24:771-779.

Janssen SA, Arntz A (1996) Anxiety and pain: attentional and endorphinergic influences. Pain 66:45-150.

Kimbrell TA, George MS, Parekh PI, Ketter TA, Podell DM, Danielson AL, Repella JD, Benson BE, Willis MW, Herscovitch P, Post RM (1999) Regional brain activity during transient self-induced anxiety and anger in healthy adults. Biol Psychiatry 46:454-465.

Kimura Y, Yoshino A, Takahashi Y, Nomura S (2004) Interhemispheric dif- 
ference in emotional response without awareness. Physiol Behav 82:727-731.

Kojima S, Goldman-Rakic PS (1982) Delay-related activity of prefrontal neurons in rhesus monkeys performing delayed response. Brain Res 248:43-49.

Kondo H, Saleem KS, Price JL (2005) Differential connections of the perirhinal and parahippocampal cortex with the orbital and medial prefrontal networks in macaque monkeys. J Comp Neurol 493:479-509.

Lang PJ, Bradley MM, Cuthbert BN (2001) International affective picture system (IAPS): technical manual and affective ratings. Gainesville, FL: Center for Research in Psychophysiology, University of Florida, Gainesville.

Lehman D, Skrandies W (1980) Reference-free identification of components of check board-evoked multichannel potential fields. Electroencephalogr Clin Neurophysiol 48:609-621.

Levy R, Goldman-Rakic PS (2000) Segregation of working memory functions within the dorsolateral prefrontal cortex. Exp Brain Res 133:23-32.

Masaoka Y, Homma I (2000) The source generator of respiratory-related anxiety potential in the human brain. Neurosci Lett 283:21-24.

Mauguière F, Merlet I, Forss N, Vanni S, Jousmaki V, Adeleine P, Hari R (1997) Activation of a distributed somatosensory cortical network in the human brain: a dipole modelling study of magnetic fields evoked by median nerve stimulation. Part II: Effects of stimulus rate, attention and stimulus detection. Electroencephalogr Clin Neurophysiol 104:290-295.

Meagher MW, Arnau RC, Rhudy JL (2001) Pain and emotion: effects of affective picture modulation. Psychosom Med 63:79-90.

Mesad S, Laff R, Devinsky O (2003) Transient postoperative prosopagnosia. Epilepsy Behav 4:567-570.

Michie PT, Bearpark HM, Crawford JM, Glue LC (1987) The effects of spatial selective attention on the somatosensory event-related potential. Psychophysiology 24:449-463.

Miller EK, Cohen JD (2001) An integrative theory of prefrontal cortex function. Annu Rev Neurosci 24:167-202.

Mobbs D, Greicius MD, Abdel-Azim E, Menon V, Reiss AL (2003) Humor modulates the mesolimbic reward centers. Neuron 40:1041-1048.

Morrison I, Lloyd D, di Pellegrino G, Roberts N (2004) Vicarious responses to pain in anterior cingulate cortex: is empathy a multisensory issue? Cogn Affect Behav Neurosci 4:270-278.

Ohara S, Crone NE, Weiss N, Lenz FA (2004) Attention to a painful cuta- neous laser stimulus modulates electrocorticographic event-related desynchronization in humans. Clin Neurophysiol 115:1641-1652.

Ohman A, Mineka S (2001) Fears, phobias, and preparedness: toward an evolved module of fear and fear learning. Psychol Rev 108:483-522.

Pascual-Marqui RD, Michel CM, Lehmann D (1994) Low resolution electromagnetic tomography: a new method for localizing electrical activity in the brain. Int J Psychophysiol 18:49-65.

Passingham D, Sakai K (2004) The prefrontal cortex and working memory: physiology and brain imaging. Curr Opin Neurobiol 14:163-168.

Persson J, Nyberg L (2000) Conjunction analysis of cortical activations common to encoding and retrieval. Microsc Res Tech 51:39-44.

Rhudy JL, Williams AE (2005) Gender differences in pain: do emotions play a role? Gend Med 2:208-226.

Sabatinelli D, Flaisch T, Bradley MM, Fitzsimmons JR, Lang PJ (2004) Affective picture perception: gender differences in visual cortex? NeuroReport 15:1109-1112.

Scherg M (1990) Fundamentals of dipole source potential analysis. In: Auditory evoked electic and magnetic fields. Topographic mapping and functional localization. Advances in audiology (Grandori F, Romani G, eds). Basel: Karger.

Singer T, Seymour B, O’Doherty J, Kaube H, Dolan RJ, Frith CD (2004) Empathy for pain involves the affective but not sensory components of pain. Science 303:1157-1162.

Stancak A, Polacek H, Vrana J, Rachmanova R, Hoechstetter K, Tintra J, Scherg M (2005) EEG source analysis and fMRI reveal two electrical sources in the fronto-parietal operculum during subepidermal finger stimulation. NeuroImage 25:8-20.

Talairach J, David M, Tournoux P (1957) Atlas d'anatomie stéréotaxique des noyaux gris centraux. Paris: Masson.

Valeriani M, Le Pera D, Niddam D, Arendt-Nielsen L, Chen AC (2000) Dipolar source modeling of somatosensory evoked potentials to painful and nonpainful median nerve stimulation. Muscle Nerve 23:194-203.

Wood JN, Grafman J (2003) Human prefrontal cortex: processing and representational perspectives. Nat Rev Neurosci 4:139-147.

Wunsch A, Philippot P, Plaghki L (2003) Affective associative learning modifies the sensory perception of nociceptive stimuli without participant's awareness. Pain 102:27-38. 